\title{
Fire and Rescue Service Reconfiguration: a case study in Nottinghamshire.
}

\author{
Peter Murphy and Kirsten Greenhalgh \\ Nottingham Business School. \\ Craig Parkin ${ }^{1}$ \\ Nottinghamshire Fire and Rescue Service.
}

Please note: In accordance with the copyright agreement with the publishers of the journal in which this article appears - this is a draft of the paper that was actually published and there will be minor changes in the published article although the substantive content and argument remains the same as this version.

\begin{abstract}
The Fire Cover Review in Nottinghamshire arises out of the Integrated Risk Management Planning process introduced in the UK by the Fire and Rescue 2004 Act,. It is intended to provide the evidence and analysis for the reconfiguration of services and the future deployment of resources in in the short, medium and long terms. Any future reconfigurations of services must be based on contemporary and comprehensive risk assessments of the areas affected.(ODPM 2004).

The need to undertake the review pre-dated the current financial crises, the general election and the need for significant reductions in public expenditure in the UK, although these circumstances made the review more challenging and politically sensitive. This paper evaluates the practical implementation of the Integrated Risk Management Planning process and the Fire Cover Review in Nottinghamshire to identify good practice and to generate recommendations for improving the process and its implementation.
\end{abstract}

Key Words Fire and Rescue, Performance Management, Emergency Response, Integrated Risk Management Planning, Service Reconfiguration.

\section{Introduction}

Fire and Rescue Authorities (FRA) in the UK are required by the 2004 Fire and Rescue Act and the current National Framework 2008-2011 to produce a local Integrated Risk Management Plan (IRMP) that sets out the authority s strategy, for reducing the commercial, economic and social impact of fires and other emergency incidents. It requires each FRA to produce a publicly available IRMP covering at least a three-year time span which, inter alia,

- "is regularly reviewed and revised and reflects up to date risk information and evaluation of service delivery outcomes

- demonstrates how prevention, protection and response activities will be best used to mitigate the impact of risk on communities in a cost effective way

- provides details of how Fire and Rescue Authorities deliver their objectives and meet the needs of communities through working with partners" (DCLG 2008, p.13)

Although the new coalition government in the UK, announced a Strategic Review of the National Framework it made it clear that it intended to retain the IRMP process and any reconfiguration of local services would therefore continue to be based upon the application of this process and its principles. Since 2004 any significant changes or service reconfigurations has had to be based upon a comprehensive contemporary risk assessment of the area affected and should use nationally accredited and approved models of risk assessment applied to robust and reliable local data and information sets.

The severe economic downturn and the medium-term reduction in public finances heralded by the Comprehensive Spending Review (CSR) 2010 has required all Fire and Rescue Authorities in England and Wales to re-examine their existing services and activities. Whilst the decision to undertake a comprehensive Fire Cover Review (FCR) in Nottinghamshire actually pre-dated the current financial crises, and political changes at local and national 
levels, the need for significant budget cuts have undoubtedly made the review more challenging for the Service and more politically sensitive for the Authority.

\section{The Nottinghamshire Fire Cover Review Project}

The Nottinghamshire FCR has four stages to its development.

1. A comprehensive re-evaluation of the existing service and prevailing risk assessments undertaken across the county. This essentially utilized the Fire Services Emergency Cover Toolkit (FSEC) supplemented by tools, techniques and information recommended by NTU which had become available since its publication.

2. The development, testing and appraisal of alternative options for changes to the service or parts of the service based upon the new countywide risk assessments undertaken in part 1.

3. The publication and public consultation on proposals for service reconfiguration; and,

4. The authoritys response to the public consultation and changes to the future deployment of the services.

Do we need the following?

The first and second stages of the FCR were completed in February and June 2011 and are considered in an earlier paper (Murphy and Greenhalgh $2011 \mathrm{a}$ ). The current paper summarises the findings of the previous paper and reports on the "consultation" stage of the project. A future paper will report on the implementation of the Fire Authority s response.

\section{Background and Context.}

Although local fire services were originally established under the Fire Services Act 1947, the Fire and Rescue Act 2004 acknowledged the wider functions and responsibilities they had gradually undertaken since 1947, redefined their roles and renamed them Fire and Rescue Services (FRSs) to reflect their actual roles and services. Over time, FRSs in the UK have had to become demonstrably proactive in preventing fires and reducing other risks to people and property rather than merely responding to fire and other emergencies as quickly as they could (ODPM 2003). At the same time the government established a new National

Framework that required FRAs to plan for, and respond to, a range of emergencies on the basis of a comprehensive and contemporary risk assessment of their areas (ODPM 2004). This fundamentally changed the basis for the configuration of their services and the deployment of their resources rather than just responding to fires :-.

"The public now expects a response to an increasing number of emergency incidents which the fire and rescue service provides or assists in providing, including:

- major transport incidents (road, rail and air);

- chemical, biological, radiological and nuclear exposures;

- severe weather conditions (especially flooding) which endanger life, property and the environment;

- explosions and collapsed structures; and

- the rescue of people trapped in buildings, vehicles, by machinery or in water." (ODPM 2003, paragraph 2.5)

While FRSs are still required to respond to fires, emergencies and other incidents the primary aim of IRMPs is to reduce and mitigate risks to individuals and communities so as to "bring about improved community safety, and to make a more productive use of fire and rescue service resources"

\section{Stage 1 The evidential base}


As part of the IRMP process the government provided each FRS with a suite of analytical tools and techniques via computer software called the Fire Service Emergency Cover (FSEC) Toolkit. This toolkit allows each fire service to undertake a risk based assessment of their area using a common approach that has been tested and independently validated. In Nottinghamshire the IRMP and the FCR were originally established to help deliver "Creating Safer Communities", the Fire Authority s strategic plan for their area and the subsequent Fire Service s operational service plan (NFRS 2010). The service wished to ensure that its resources are efficiently and effectively deployed, at the point of need and appropriately configured to avoid, minimise or mitigate all fire and safety risks to the community in the short, medium and long terms. NFRS commenced the review prior to the financial crises, the national and local political changes, and the significant reductions in public expenditure, although these external changes undoubtedly made the review more challenging for the service and more politically sensitive for the Authority.

NFRS used the latest and most appropriate analytical tools and information available for the project and constructed a new evidential base that enabled them to undertake a robust, comprehensive and consistent review of the individual and community risks at various levels across the county (Murphy and Greenhalgh 2011 a). However the configuration of services and deployment of resources had largely arisen from the historical remit and previous objectives for the service, which had now been superseded. As a result the data, information, standards and benchmarks upon which the previous risk assessments were based were no longer "fit for purpose" and this resulted in a less than optimal pattern of resource deployment for either current needs or anticipated future patterns of risk across the county

The historical standards and the former Key National Indicators, were based primarily on incident response times, which have now largely been superseded. As part of Stage one of the project the risk assessment data for Nottinghamshire was cleansed, improved, updated and supplemented, to meet the requirements of the FCR. These improvements were greatly facilitated by significant improvements to the mapping and computer technology available to the service and the development of the FSEC toolkit . The current exercise would not have been feasible without this new technology.

\section{Stage 2 Reconfiguration Options}

The evaluation of stages one and two not surprisingly found that in a period of financial constraints and reduced resources, decisions on service reconfigurations became overtly politically contested, and continue to generate considerable public interest. This meant that finalizing the proposals for the public consultation stage took longer and was more difficult than anticipated despite the urgent need for productivity improvements and efficiency savings (Murphy and Greenhalgh 2011a),.

NFRS produced its report on the evidential base, the data collection and the development of options in February but the Fire Authority took until June 2011 to produce its conclusions (NNFRA 2011). This may have been partially explained by the fact that the review produced a large number of recommendations or potential improvements to many parts of the service across the county, and that these recommendations had many interdependencies and multiple consequences depending on the mix of recommendations. However, throughout this period a number of campaigns by stakeholders and pressure groups, mostly based around changes affecting individual fire stations, became increasingly politically active (Nottingham Local News 2011).

Nevertheless in June the CFO presented three draft strategic options to the Authority for changes to the configuration and deployment of services and resources across the county (NFRS 2011). As his report explained

${ }^{2}$ For its risk mapping, Nottinghamshire used the more sophisticated Merseyside FRS model rather than the earlier Lancashire FRS model 


\begin{abstract}
"Although many communities will view their local fire station as being "their" provision, the reality is that in terms of a county-wide service, management has to take a holistic view. It is therefore not feasible to present to the Fire Authority a series of recommendations, or indeed options, around individual units. The reality is that for every action there is a consequent reaction in the provision of fire cover. Changing the availability of one appliance or station will have a knock on effect to the next nearest appliance or station. This is why it is so important to consult fully on any changes" (NFRS 2011 p.3).
\end{abstract}

The CFO presented three options as "packages" and identified a preferred option. All three options had over 30 (often inter-related) parts to their "package" and to assist the decision and to facilitate early implementation, the CFO broke these recommendations into three categories for each of the three potential options. Thus potential recommendations or changes involving "management capacity" were differentiated from proposals or recommendations for "stations and appliances" and from other "supporting considerations" which dealt with issues such as the non uniform staff structure, consequential training requirements, estate review, and changes to the Fire Control Centre. The Fire Authority based the public consultation exercise on a variant of one of these options (NNFRA 2011a).

\title{
Stage 3 Public Consultation and Engagement
}

The consultation exercise was the largest ever undertaken by NFRS with an unprecedented range of activities, levels of interest and number of responses. It was advised and assisted by independent consultants, Opinion Research Services (ORS), who are accredited by the government for the implementation of public consultations arising out of the IRMP process.

The consultation exercise commenced on $1^{\text {st }}$ August and was originally intended to run for a period of 12 weeks, to finish on the $23^{\text {rd }}$ October 2011. However the Authority decided to extend the consultation period by a further three weeks due to the unprecedented level of interest, and the volume of representations being received about their proposals..

By adopting a wide ranging, inclusive and open approach to the public consultation, the FRS and the FA reduced the risk of future challenges to the process and decisions from Judicial Review. Judicial Review challenges having been both successfully and unsuccessfully lodged against the process as well as the content of reconfiguration proposals elsewhere (Royal Berkshire FRA 2009).

\section{Methodology}

\section{a) The evidential base and option development}

NTU were appointed as independent consultants on the FCR with a remit to review the adequacy and objectivity of the evidential base and to provide assurance as to the process undertaken by NFRS. Their approach to the consultancy was from a functionalist perspective with the relationship between the client and consultant being considered as an arms-length, contractual and independent relationship where the needs of the client come first (Werr \& Styhre 2003)..

The authors investigated the background policy and technical guidance developed for the IRMP (DCLG 2008), to establish an understanding of the multivariate factors affecting the analysis of risk across Nottinghamshire and therefore factors and data to be examined within the FCR. In addition to the legislative frameworks and the national standards and requirements, further key mutually interdependent variables and factors creating and responding to risk across the county were identified.

The authors advised and appraised all three successive versions of the Community Profiles and the final versions of these documents are all now publically available within the publications section of the Nottinghamshire Fire and Rescue Service web site. 
To arrive at its complete assessment it was also necessary to investigate and coordinate risk assessment and the deployment of current and future resources across counties boundary. This was particularly important in Nottinghamshire s case because of the existence of several stations and services situated close to the county boundary but located within the jurisdiction of other Fire and Rescue Authorities.

\section{b) Public consultation and engagement}

The methodology adopted for the analysis of the public consultation stage had two key elements

- An appraisal of the process and techniques used by other FRSs in their IRMP processes, and

- An analysis of the responses received to the NFRS consultation and engagement.

In the UK each FRS is clustered into one of five national „family groups. These family groups are derived from the Chartered Institute of Public Finance and Accountancy,,S „Nearest Neighbour model. These nationally prescribed group clusters were established on the basis of the services being "most similar in terms of geographic and demographic areas. NFRS is within family group four, along with 15 other English FRSs, the Northern Ireland FRS and the South Wales FRS. The authors have used this family group as the sample for establishing the comparative analysis in terms of the IRMP process, the level of detail provided to the public, the consultation process undertaken. Each one of the FRSs has a publically available, and easily accessible, IRMP document apart from Avon FRS whose latest documentation on the IRMP relates to the earlier 2008-2011 period. The analysis therefore relates only to the other seventeen FRSs within group four.

This took the form of ethnographic content analysis exploring underlying themes in the documentation available to the public via the organisation websites. In ethnographic content analysis, "Categories and variables initially guide the study, but others are allowed and expected to emerge during the study (Altheide 1996, p.16). The variables analysed in this case being: the IRMP period, the document title, the level of changes considered and detailed to the public, the consultation period, the consultation questions and FRA approval.

\section{Comparative analysis and findings}

The IRMP timespan differs for each FRS ranging from 2011/12 to 2011/20. Only four of the seventeen FRSs, including NFRS, have detailed option plans with specific station/areas cited. Each of the services with specific detail provided a three month consultation period; two of these were still out for consultation at the time of our study whilst two have been out to consultation, amended and have received authority approval. One FRS completed a review in 2010 which proposed no change to the station locations within its area, and received authority approval for its recommendations. This FRS then considered changes to crewing methods.

The remaining twelve FRSs were all undertaking detailed service cover reviews to generate options to address IRMP requirements and realign resources to the areas of greatest risk but had not yet provided the specific details relating to stations/areas, although some of these FRSs did cite individual stations for individual proposals. An example of the phraseology being used within the online surveys is, "Do you support the approach to review and potentially change the locations of existing stations and to review the response times of appliances to ensure a more effective emergency response to communities?" (Cheshire FRS 2011)

Unlike Nottinghamshire, the vast majority of the FRSs are specifically highlighting the reduction in public funding as the prevailing factor for reviewing the fire cover in their area, for example one was, "researching, and where appropriate proposing and consulting on, options for reducing costs to the public without reducing frontline services" (Humberside FRS 2011, p.11). 
In summary, NFRS was undertaking a detailed, comprehensive and strategic review based upon a fundamental re-evaluation of individual and collective risks across its area. This was motivated by a desire to achieve the optimal pattern of resource deployment to mitigate these risk both now and in the future, driven by the new IRMP process. Part way through the process, as a result of the economic downturn, it had inevitably to deal with the financial restriction but this was not its original raison d etre. Even at this stage had the NFRS or the FA wished to adopt a strategy driven by cost reduction, then they would not have supported the CFO s preferred option but would have chosen the option specifically designed around such a strategy. Although previous service reconfigurations in other FRSs resulted from the IRMP process these generally predated the significant financial constraints of CSR 2010. The on-going post 2010 reviews have generally taken cost constraints as their primary motivator for service reconfiguration.

\section{Analysis and findings from the consultation response}

NFRS presented their proposals in three policy "clusters", namely proposals for "stations and appliances"; "managerial capacity" and other implementation or "consequential considerations". They also identified and analysed the response in relation to four key stakeholder groups "the general public and individuals"; "businesses"; "key delivery partners" and "representative groups".

Insert diagram?

\section{Stations and appliances}

The formal consultation document (NFRS 2011a) concentrated entirely on the issue of proposed changes to stations and appliances although the public meetings and other initiatives also discussed the other issues. All but one question in the formal questionnaire referred to station or appliance specific changes, so not surprisingly these issues were the subject of the vast majority of responses from the public. Misinformation also overwhelmingly related to these issues, although concerns were alleviated when accurate information was made available through the NFRS website or at the formal consultation meetings

it is hard to escape the conclusion that there is in fact relatively little opposition to the Fire Cover Review's draft proposals across Nottinghamshire. This impression is reinforced by NFRS' experience in its local meetings and on-street information events across the county - and confirmed by the outcomes of the questionnaire survey - in which seven out of nine proposals were approved by absolute majorities (ORS 2011, p.9).

There were surprisingly few responses from business and other "non-domestic responders both before and after the extension of consultation period despite the extra attempts to engage this sector. It is interesting to note that NFRS is already actively examining how this can be improved for the IRMP 2013 review.

Key partner responses were also generally supportive. This was in contrast to previous reductions of services often resulted in objections to the proposals. However, in the prevailing financial environment, key stakeholders across the emergency services or in the local strategic partnership (LSP) tended embraced the changes and perceived the reconfiguration as an opportunity to rationalise public owned property or as a catalyst to reconsider the configuration of their own related services.

Essentially the representative bodies took a defensive stance to their members vested interest. The response from these bodies predictably depended upon whether the proposals affected whole-time stations and appliances or retained staff stations and appliances.

\section{Managerial capacity}

The public were generally unaware of these issues and only tended to comment after an interchange with a „formal representative of the service. They were however generally 
supportive when they were informed that managerial capacity would be reduced disproportionately over front-line capacity. "At least you ve got your priorities right" encapsulates the universal response.

As these proposals potentially impact upon emergency preparedness, planning integrated responses and collaborative working, one might have expected that there would have been a critical reaction. However, again the key stakeholders, acknowledging the shrinking resource envelope, and recognising the robust process that the service had been undertaking, had surprisingly few comments and virtually no criticisms.

Surprisingly, the representative bodies took very little interest in these proposals even though they potentially affected some of their members.Similarly the business community were surprisingly uninterested in these issues, their overriding consideration being the potential impact on operational response times and level of risk to their individual premises.

\section{Implementation or consequential considerations}

These considerations were undeterminable at the consultation stage of the process since they are entirely dependent upon the outcome of the consultation and the subsequent decisions. They are essentially matters that need to be addressed as part of the implementation of the revised service deployment. These „additional consequential considerations remained the same within all the options and applied whatever reconfiguration strategy was chosen. These proposals covered issues such as service structure, estate, training, the impact on the Control Centre and amendments to rotas and duty systems.

The public were generally unaware or uninterested in these proposals. There were also relatively few comments from Key stakeholders. The only significant issue that was raised was the future of the regional Fire Control Centre and this was largely due to a misunderstanding relating to how this had been funded and which agency would be responsible for future liabilities.

The business community like the general public and the key stakeholders had almost no comments on these issues although the representative bodies, not surprisingly, took a keen interest in the potential human resource impact of these implementation matters.

\section{Some wider issues}

When evaluating the process it was apparent that the open and transparent approach to stages one and two of the project contrasted with the more limited approach to information sharing that characterises most of the other on-going IRMP exercises scrutinised. Nevertheless in our view it was highly successful and retrospectively widely appreciated.

The publication at the earliest opportunity, on the unrestricted part of the NFRS website, of all the data and evidence used to arrive at recommendations and decisions for Stages 1 and 2 of the FCR, clearly reduced early speculation and suspicion among key stakeholders. Unions and local pressure groups both changed their positions and their tactics as a result of this information being put into the public domain. Early publication of schedules and timetables of when information would be publically available also helped "manage" the pre-publication speculation and reduced the number and impact of campaigns based upon deliberate misinformation, rumour or gossip. Although it did not completely eradicate all mis-information a number of these campaigns stopped expanding and quickly fizzled out when potential adherents accessed the publicly available data and information - often changing their position and advising the NFRS of the mis-information (Murphy et al 2011).

The challenges, contributions, objections and responses to the consultation were conducted and/or communicated through a much wider range of communication channels than has ever been the case in the past. This results from the proliferation of these communication channels and the easier access and increased use of mobile technology and the internet. A 
number of "campaigns" were established around individual proposals for changes to services and/or the retention or closure of stations. Some groups established websites for the purpose of campaigning and generating responses (Nottinghamshire Local News 2011). Similarly, some of the representative bodies specifically created websites encouraging people to, inter alia, respond to the consultation, although they were not formally part of the consultation exercise and were unsolicited by either the NFRS or the FA. Although these representative bodies were key stakeholders they inevitably had vested interests and their own objectives, some of which are different to those of the NFRS, the FA and to each other.

The decision to produce a preferred "package" of proposals, and to make explicit the interdependencies between proposals were these exist, rather than a series of individual proposals, undoubtedly led to a more considered and informed response to the FCR than to previous NFRS consultations.

The early release of detailed information on both the process and the evidential base was generally welcomed and proved very effective. The use of independent consultants for quality assuring the evidential base and the consultation process itself was almost universally acknowledged to have improved the process in terms of its objectivity. It also gave reassurance to the FA members, the senior management of the service, the project team and key stakeholders. The FA and the FRS regarded the appointment of the independent consultants as not only good practice but an extremely cost-effective use of their resources for which they have a duty of „Best Value. The only reservation was amongst some members of the FA who retrospectively questioned the use of consultants when they received the feedback from the consultation process and some responses caused controversy.

\section{Conclusions, lessons and recommendations - to be expanded and completed prior to the research publication seminar}

Conclusions on the evidential base and IRMP process

- To be adapted from working paper 1

\section{Conclusions on the FCR exercise such as}

- The level of detail provided and the transparency of the process tended to be far greater than similar exercise undertaken in our comparative analysis due to the proactive nature adopted by NFRS.

- The general era of austerity and its constraints on public expenditure have manifestly influenced and contextualised the attitude and response of public sector providers and the business community, within the key stakeholders, and to a lesser extent the public and representative bodies

- Conclusions relating to the validity and robustness of the consultation process

\section{Recommendations for the future}

- There must be a clear distinction between the FRSs responsibility, to provide accurate information and robust advice, and the FA s ultimate responsibility, to determine priorities and take key strategic decision on long-term resource planning ( $r$

- Recommendations for improving engagement in Nottinghamshire.

- Recommendations for the medium and long-term strategy for FCR in Nottinghamshire

- Process recommendations and improvements for all FCRs

- Recommendations for future research 


\section{References}

Altheide D, L. (1996) Qualitative Media Analysis. A Sage University Paper, Sage publications California USA

Cheshire FRS, 2011. IRMP 9 Consultation: Residents' survey.

http://www.surveymonkey.com/s.aspx?sm=7jecYIPamZw1ESU\%2bU712Hmw5OBpEO3stkZ \%2bJXebZOlw\%3. [accessed on 04/11/11]

DCLG 2008. IRMP Steering Group Integrated Risk Management Planning: Policy Guidance, HMSO, London http://www.communities.gov.uk/documents/fire/

DCLG 2008. Fire and Rescue Service National Framework 2008-11, HMSO, London. ISBN: 978-1-4098-0013-2

http://www.communities.gov.uk/documents/fire/doc/nationalframework200811.doc

DCLG 2010. Leading a lean and efficient fire and rescue service, Fire Minister Bob Neill s speech to Fire and Rescue 2010 Conference, Harrogate, England, http://www.communities.gov.uk/speeches/fire/frsconference2010

Humberside FRS, 2010. Strategic Plan: 2011-2014,http://www.humbersidefire.gov.uk/fireservice/documents

Konukcu, S and Bouchlaghem, D. 2010. A holistic approach to fire risk management for buildings in the UK. - Department of Communities and Local Government, IRMP Project Papers. Available at_http://irmp.lboro.ac.uk/publications.php

Murphy, P. and Greenhalgh, K., 2011. Creating the new national framework for the Service. FIRE, Vol. 104 October 2011 pp.40-41 (1342).

Murphy, P. and Greenhalgh, K., 2011a. Fire and Rescue Service Reconfiguration in an Era of Austerity, - a case study of the Fire Cover Review in Nottinghamshire. The Public Administration Committee (PAC) Annual Conference, International Conference Centre, Birmingham, $6^{\text {th }}$ September 2011.

Murphy, P., Greenhalgh, K. and Coleman, P., 2012 (forthcoming): "The development of new support and intervention arrangements for Fire and Rescue Services". To be presented at the forthcoming Alternative Futures Conference at Nottingham Trent University $22^{\text {nd }}$ February 2012.

Murphy, P., Greenhalgh, K. and Parkin, C, 2011. The Fire Cover Review in Nottinghamshire: Working paper 2 - Interim findings of stage 3 of a Fire and Rescue Service Reconfiguration. Fire Related Research and Developments (RE11) Annual Conference, National Fire Service College, Gloucestershire, $17^{\text {th }}$ November 2011.

Nottingham Local News 2011. Bridgford may lose its Fire station. Available at: http://www.nottinghamlocalnews.com/2011/01/bridgford-may-lose-its-fire-station/. [Accsessed on $4 / 11 / 11$ ]

Nottinghamshire and City of Nottingham Fire and Rescue Authority 2011. Fire Cover Review Report of the Chief Fire Officer 18 February 2011

http://open.nottinghamcity.gov.uk/comm/download3.asp?dltype=inline\&filename=46945/1102 25FINAL2FCR.pdf

Nottinghamshire and City of Nottingham Fire and Rescue Authority 2011a. Options for addressing the outcomes of the Fire Cover Review 2010 Report of the Chief Fire Officer 24 June 2011 at http://open.nottinghamcity.gov.uk/comm/agenda.asp?CtteMeet/D=4132 
Nottinghamshire and City of Nottingham Fire and Rescue Authority 2011b. Fire Cover Review Consultation Process: Report of the Chief Fire Officer to Policy and Strategy Committee October 2011 available at

http://open.nottinghamcity.gov.uk/comm/download3.asp?dltype=inline\&filename=49202/FINA LConsultationProcess.pdf

Nottinghamshire Fire and Rescue Service 2010. Nottinghamshire Fire and Rescue Service Plan 2010-2013 available on http://www.notts-fire.gov.uk/Documents/NFRS Service Plan 20102013 full document.pdf

Nottinghamshire Fire and Rescue Service 2011. Options for addressing the outcomes of the Fire Cover Review Report of the Chief Fire Officer $24^{\text {th }}$ June 2011 http://www.notts-fire.gov.uk/Documents/FCR options and full report.pdf

Nottinghamshire Fire and Rescue Service 2011a. Your service, Our Vision, Fire Service Review Consultation Document http://www.notts-fire.gov.uk/A51BBFF08670491A9EB906F5978A6AB8.asp

ODPM 2003. Our Fire and Rescue Service White Paper Cmnd 5808 London Stationary Office

ODPM Minister 2004. The Fire and Rescue National Framework 2005/06 London Stationary Office

Opinion Research Services, 2011. NOTTINGHAMSHIRE FIRE AND RESCUE SERVICE FIRE COVER REVIEW : An Independent Report of the Consultation. Swansea

Royal Berkshire Fire and Rescue Authority 2009. Judicial Review backs Royal Berkshire Fire Authority. Available at: http://www.rbfrs.co.uk/press releases09/17-02-2009.pdf

Werr, A. and Styhre, A., 2003, Management Consultants - Friend or Foe? Understanding the Ambiguous Client-Consultant Relationship. International Studies of Management and Organization Issue: Volume 32, Number 4 / Winter 2002 pp.: 43 - 66

\section{Contacts}

Peter.murphy@ntu.ac.uk Kirsten.greenhalgh@ntu.ac.uk

01158488092 01158488017

Methodology Stages 1 and 2 refers to

Werr \& Styhre 2003

DCLG 2008a, 2008b, 2008c, 2008d, 2008e, 2008f, 2008g, DCLG 2009 